Ciencia y Educación, Vol. 4, No. 3, septiembre-diciembre, 2020

ISSN (impreso): 2613-8794 • ISSN (en línea): 2613-8808

DOI: https://doi.org/10.22206/cyed.2020.v4i3.pp7-25

\title{
Gestión y conceptualización de las interacciones con el entorno en universidades argentinas
}

\author{
Management and conceptualization of interactions with \\ the environment in Argentine universities
}

Mariana Eva Di Belloa ORCID: 0000-0002-1128-7006

Lucía Ana Romero ${ }^{\mathrm{b}}$ ORCID: 0000-0002-3211-5262

Fernanda Andrea Soca ${ }^{c}$ ORCID: 0000-0003-1432-4762

Pablo Gabriel Sánchez Macchioli ${ }^{\mathrm{d}}$ ORCID: 0000-0002-9022-8812

Recibido: 29/02/2020 • Aprobado: 24/04/2020

Cómo citar: Di Bello, M. E., Romero, L. A., Soca, F. A., \& Sánchez Macchioli, P. G. (2020). Gestión y conceptualización de las interacciones con el entorno en universidades argentinas. Ciencia y Educación, 4(3), 7-25. Doi: https://doi.org/10.22206/ cyed.2020.v4i3.pp7-25

\section{Resumen}

El trabajo analiza de forma comparada concepciones de funcionarios universitarios e instrumentos de gestión de la relación con el entorno comunitario en cuatro universidades nacionales argentinas: Universidad Nacional del Sur (UNS), Universidad Nacional de Mar del Plata (UNMDP), Universidad Nacional de Quilmes (UNQ) y Universidad Nacional Arturo Jauretche (UNAJ). Se utilizan técnicas de revisión de documentos institucionales y análisis interpretativo de entrevistas. Los hallazgos encontrados revelan una jerarquización de espacios organizacionales y la generación de nuevos instrumentos de

\begin{abstract}
This work analyses, in a comparative way, university' staff conceptions and management devices that promote interactions with the community environment in four Argentine national universities: Universidad Nacional del Sur, Universidad Nacional de Mar del Plata, Universidad Nacional de Quilmes and Universidad Nacional Arturo Jauretche. The analytic procedure entails institutional documents review along with interpretive analysis of interviews. Findings reveal a hierarchy of organizational spaces and the generation of new policy and management instruments for the environment interaction. Three main
\end{abstract}

\footnotetext{
a Consejo Nacional de Investigaciones Científicas y Técnicas (CONICET). Instituto de Estudios sobre la Ciencia y la Tecnología- Universidad Nacional de Quilmes (IESCT-UNQ). Docente Universidad Nacional de La Plata (UNLP). Argentina.

Correo-e: mariana.di.bello@unq.edu.ar

b Consejo Nacional de Investigaciones Científicas y Técnicas (CONICET). Instituto de Estudios sobre la Ciencia y la Tecnología- Universidad Nacional de Quilmes (IESCT-UNQ). Docente Universidad de Buenos Aires (UBA). Argentina.

Correo-e: laromero@unq.edu.ar

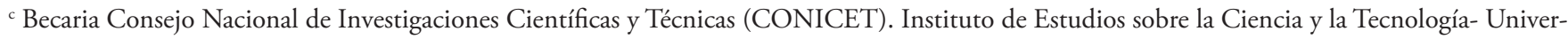
sidad Nacional de Quilmes (IESCT-UNQ). Argentina.

Correo-e: fsoca@uvq.edu.ar

d Becario Agencia Nacional de Promoción de la Investigación, el Desarrollo Tecnológico y la Innovación (Agencia). Instituto de Estudios sobre la Ciencia y la Tecnología- Universidad Nacional de Quilmes (IESCT-UNQ). Argentina.

Correo-e: psanchezmacchioli@uvq.edu.ar
} 
política y gestión para la interacción con el medio. Se identifican tres modalidades predominantes de interacción con el medio: a) la universidad como sede; b) la participación en polos científico-tecnológicos y c) el establecimiento de nodos.

Palabras clave: universidad; entorno; gestión; concepciones; Argentina.

\section{Introducción}

La educación superior es concebida, cada vez más, como un vehículo para el desarrollo de la región en cuyo tejido social y económico está implicada (Goddard y Chatterton, 2003). Sin embargo, la forma que asume este fenómeno varía mucho entre regiones y estilos de universidades (Pinheiro, Benneworth y Jones, 2012). En países como Argentina, muchas universidades están ubicadas en regiones o localidades cuyas posibles demandas de conocimientos no se asocian a actividades innovadoras en el ámbito productivo, sino a la resolución de problemáticas sociales. En ese contexto el trabajo busca, a partir de un estudio de casos, analizar los instrumentos de gestión y las concepciones que los funcionarios universitarios tienen sobre el rol de la universidad en su entorno regional.

El artículo se estructura de la siguiente forma: en el primer apartado se realiza una revisión de la literatura que en los últimos años ha abordado el fenómeno de la emergencia e institucionalización de la llamada "tercera misión" de la universidad, y se describen, para el caso argentino, las políticas públicas recientes orientadas a fomentar los lazos entre las universidades y sus entornos. Luego se caracteriza brevemente a las cuatro universidades y a sus entornos geográficos y se realiza un análisis de los instrumentos institucionales y discursos del staff universitario. Finalmente, se sistematizan, de forma comparada, los hallazgos encontrados. types of universities-socio-economic setting relationships are identified: a) the university as a site; b) the participation on scientific-technological poles and c) the settling of nodes.

Keywords: university; socio-economic setting; management; conceptions; Argentina.

\section{Cambios recientes en la "tercera misión" de la universidad}

A nivel global, durante las últimas décadas, hemos asistido a un crecimiento sostenido del interés por comprender las características y los procesos de institucionalización de la denominada "tercera misión" de la universidad. Durante las décadas de 1980 y 1990, el acento analítico y la preocupación de los hacedores de política pública y organismos multilaterales se posó, casi unilateralmente, sobre las posibilidades de vinculación entre las instituciones generadoras de conocimientos científico-tecnológicos y los sectores productivos. Desde visiones optimistas o críticas, varios autores analizaron este proceso atendiendo a sus múltiples aristas. Los enfoques más difundidos y citados corresponden al modelo de la Triple Hélice (Etzkowitz y Leydesdorff, 2000), los Sistemas Nacionales o Regionales de Innovación (Dosi 1999; Edquist 1997; Lundvall, 1992; Nelson, 1993), Nuevo Contrato Social entre ciencia y sociedad (Guston y Keniston, 1994), Modo 2 de Conocimientos (Gibbons et al., 1997), Universidad Emprendedora (Clark, 1998), Capitalismo Académico (Slaughter y Leslie, 1997) y Estado Evaluador (Neave y van Vught, 1991).

Aunque esos marcos teóricos y los fenómenos a los que refieren siguen vigentes, a partir de los años 2000 la noción de tercera misión de la universidad es repensada. Este replanteo del tema alude fundamentalmente a incluir en el análisis vinculaciones 
que las universidades — desde siempre- entablan con sus entornos, con actores variados no solo de los ámbitos productivos, sino sociales, políticos y culturales. En este sentido, gran parte de los trabajos reconocen el sesgo economicista de los primeros enfoques y proponen evitar una mirada reduccionista del rol de las universidades en sus entornos (Harding, Laske y Scott, 2007). Esto significa, en términos generales, no acotar el análisis a los procesos de vinculación asociados a innovaciones científico-tecnológicas o a la valorización del conocimiento en el mercado e incluir el variado rango de actividades donde la universidad se ve involucrada aportando conocimientos, recursos o capacidades (Laredo, 2007; Kruss Visser, Aphane y Haupt, 2012).

Este renovado interés por estudiar la tercera misión de la universidad se expresa en una serie de acciones de política pública de educación superior, tanto a nivel de las naciones o regiones supranacionales como de la política universitaria (Arbo y Benneworth, 2007; Enders, Boer y Westerheijde, 2011; Jacob, Sutin, Weidman y Yeager, 2015; Kwiek, 2013) y formación de consorcios o redes regionales de universidades (Hoyt y Hollister, 2014).

$\mathrm{Si}$ bien se puede argumentar que se trata de un fenómeno global, las diferentes características y trayectorias institucionales y contextos sociopolíticos de los distintos sistemas nacionales de educación superior otorgan particularidades locales al proceso de emergencia e institucionalización de la "tercera misión" de la universidad. Sin embargo, como sucede con otros tópicos analíticos, en la literatura de mayor difusión prevalecen los marcos conceptuales y los estudios empíricos generados en países desarrollados para comprender sus propias realidades.

De allí proviene la revisión del modelo de la triple hélice y la propuesta de reemplazo por el modelo de cuádruple hélice, que incorpora como cuarto actor o hélice a los sectores civiles, a los usuarios de los conocimientos (Arnkil, Järvensivu, Koski y Piirainen, 2010). También surgió, con base en estudios de caso realizados en Australia, la conceptualización de dos tipos ideales de universidad en relación a su interacción con el entorno: un tipo de universidad genera- tiva, capaz de proveer conocimientos que dinamicen los procesos de innovación de las regiones; y un tipo desarrollista (developmental), donde la universidad asume un vínculo con el entorno a partir de integrar redes con actores locales, suministrando recursos humanos y cognitivos para resolver demandas o problemas específicos (Gunasekara, 2006).

Otra serie de trabajos originados en Europa analizan los cambios recientes en la tercera misión de la universidad en términos del advenimiento de una misión regional (Pinheiro et al., 2012) o un compromiso regional (regional engagement) de la universidad (Gál y Zsibók, 2011). Los trabajos enmarcados en esta perspectiva proponen comprender el proceso de emergencia e institucionalización de la misión regional de la universidad analizando tanto los cambios en la organización universitaria como las transformaciones que han ocurrido en las demandas sociales hacia la universidad (Benneworth, Pinheiro y Sánchez-Barrioluengo, 2016; Pinheiro et al., 2012).

Una variante del enfoque del compromiso regional de la universidad son las propuestas de la universidad cívica (civic university) (Goddard, Hazelkorn, Kempton, y Vallance, 2016) y la "universidad como institución insignia” o "pilar" de la localidad o región (university as an anchor institution) (Goddard, Coombes, Kempton, y Vallance, 2014). Estos enfoques entienden que las instituciones de educación superior son muy reconocidas por la comunidad que las circunda y que generan externalidades positivas porque son capaces de actuar como pilar de otras organizaciones o "anclar" actividades económicas en una región. En contraste con un modelo de universidad tradicional o "no cívica" (un-civic), la universidad cívica se caracteriza por ubicar a la tercera misión como un objetivo central de la institución que atraviesa todas sus funciones, por tener una identificación local y un sentido de pertinencia de sus actividades y permitir una amplia participación de actores extra-académicos en decisiones institucionales (Goddard y Kempton, 2016).

Otro abordaje usado para analizar la tercera misión universitaria, se apoya en el concepto de innovación social, entendido como la generación de 
acciones y procesos de cambio creativos o novedosos en el ámbito de las funciones sustantivas de la universidad para hacer frente a problemáticas sociales del entorno. (Mulgan, Tucker, Ali y Sanders., 2007; Villa Sánchez, 2014).

Es conocido que América Latina posee una larga tradición en lo que se refiere a la "tercera misión" de la universidad. Ciertamente, su incorporación en términos de la función de "extensión universitaria” fue uno de los pilares del movimiento reformista iniciado en Córdoba, Argentina, en 1918 y expandido a toda la región (Tünnermann, 2008). Otro hito importante en términos de pensar el aporte de las universidades, o más precisamente, de los conocimientos científicos y tecnológicos al desarrollo local, puede encontrarse en los autores que conforman el denominado pensamiento latinoamericano en ciencia, tecnología y desarrollo. Pero, aunque con orígenes previos, fue particularmente a partir de la década de 1990 cuando se generaron numerosos trabajos en la región sobre el rol de las universidades en el desarrollo productivo - usualmente desde la perspectiva de la triple hélice y de los Sistemas Nacionales de Innovación-, enfoque muy usado asimismo en la política pública. Algunos trabajos pioneros, basados en estudios de caso mostraron también las dificultades para lograr la vinculación de la academia con los sectores productivos o su excepcional superación en algunos casos considerados "exitosos" (Schwartzman, 2008; Sutz, 1996; Tenti Fanfani, 1993; Vessuri, 1995). Otro amplio abanico de trabajos analizó este fenómeno a la luz de los cambios en la organización y misión universitaria y en la profesión académica (Gentile y Levy, 2005; Naishtat, García Raggio y Villavicencio., 2001; Mollis, 2003; Schugurensky, 2007).

Si bien la preocupación por lograr mecanismos eficaces de vinculación entre las universidades y los sectores productivos continúa vigente y sigue siendo un desafío, en las últimas dos décadas presenciamos - con vaivenes derivados de las distintas coyunturas políticas - un proceso de revalorización del rol social de las universidades y un reconocimiento de que son instituciones que atienden una amplia variabilidad de demandas de la sociedad (Arocena y Sutz
2015; Di Bello y Romero, 2018a, 2018b; Fernández Lamarra, 2009; García Guadilla, 2010; Lischetti, 2013; Mato, 2009; Naidorf, 2009; Riquelme, 2008; Romero, Buschini, Vaccarezza y Zabala 2015; Storni, 2005; Zangrossi, 2013). El reconocimiento del rol de las universidades como agentes de transformación social de las comunidades donde se asientan cobra fuerza, asimismo, en el discurso de actores universitarios de la región, quienes progresivamente adoptan conceptos como responsabilidad social universitaria, diálogo de saberes (Vallaeys, 2006; Appe, Rubaii, Lippez de Castro y Capobianco, 2017), co-construcción de conocimientos o compromiso social universitario (Pérez, Cecchi, Lakonich y Rotstein, 2009; Cecchi, Pérez y Sanllorenti, 2013).

Esto sucede en paralelo a un proceso de cambio a nivel de la política pública y universitaria. En Argentina en particular, durante los años 2000, se crearon una serie de espacios institucionales y acciones de política de educación superior orientadas a fortalecer la relación de las universidades con el medio, por fuera de una lógica de mercado. Así se creó el "Programa Nacional de Voluntariado Universitario" en 2002, dentro de la Secretaría de Políticas Universitarias del entonces Ministerio Nacional de Educación, Ciencia y Tecnología, que volcó una significativa cantidad de recursos en las universidades para proyectos que tuvieran una intervención en los territorios. El Ministerio de Educación, Ciencia y Tecnología promovió la realización de Congresos Nacionales de Extensión Universitaria y Jornadas Nacionales de Extensión Universitaria desde 2002 mientras que el Consejo Interuniversitario Nacional (CIN) tuvo un importante rol en el impulso y redefinición de la extensión universitaria.

Otros programas promovieron la vinculación de la universidad con la educación de nivel medio y fomentaron vocaciones profesionales y científicas en jóvenes provenientes de sectores sociales tradicionalmente excluidos de la educación superior. Tanto esos programas como los denominados "Cooperativismo y Economía Social”, "Vinculación tecnológica” y "Universidad, Estado y Territorio" resaltaron el carácter territorial de los vínculos de la univer- 
sidad. Asimismo, se crearon becas estudiantiles y se amplió el número de beneficiarios, se originó el Plan de Finalización de Estudios Primarios y Secundarios "FinEs" orientado a otorgar incentivos a los jóvenes para rendir materias pendientes del nivel secundario (Marquina y Chiroleu, 2015) y el Programa de Respaldo a Estudiantes Argentinos (PROG.R.ES. AR) dirigido a jóvenes en situación de vulnerabilidad que desearan finalizar sus estudios secundarios e iniciar o continuar con estudios superiores. Por su parte, en los últimos años, diversas universidades públicas generaron iniciativas tendientes a fortalecer las relaciones con el entorno en el cual se asientan. Por ejemplo, varias universidades nacionales han creado recientemente Consejos Consultivos Comunitarios o Consejos Sociales, mientras que otras crearon normativas para la institucionalización de Prácticas Socio Comunitarias en los planes curriculares como requisito para obtener el título de graduación (Cecchi et al., 2013).

Un elemento que sobresale en los programas e instrumentos de financiamiento del período es el uso del concepto de territorio (Sturniolo y Naidorf, 2007). La noción de territorio cuestiona una larga tradición de la extensión concebida como un vínculo de la academia con la sociedad —entendida generalmente como ser nacional- La apelación territorial, por el contrario, delimita un espacio geográfico e identitario: ya no es la sociedad toda la que se beneficiará de la difusión de conocimientos, sino una comunidad específica, definida a partir de un conjunto compartido de reglas, valores y prácticas determinadas (Di Bello y Romero, 2018a).

\section{Metodología}

Atendiendo a las características del objeto de estudio - un fenómeno poco institucionalizado, la existencia de amplia heterogeneidad entre las instituciones analizadas-, la estrategia metodológica asume un diseño cualitativo y flexible (Hammersley y Atkinson, 1994). Uno de los elementos distintivos de este tipo de investigación es su carácter inductivo, emergente. Se busca en esta forma de ejercer la investigación crear conceptos a partir de los datos encontrados antes que verificar hipótesis teóricas mediante un trabajo empírico (Mendizábal, 2006). En este sentido, si bien se asume que todas las perspectivas y conceptos reseñados son útiles para pensar el problema, no se utiliza un parámetro teórico.

Los casos fueron seleccionados en función de sus diferentes contextos de creación y ubicación para la realización de una investigación más amplia que busca identificar y definir modalidades de interacción de universidades con sus entornos.

Este estudio persigue responder tres preguntas de investigación: 1) ¿cómo se expresa el vínculo con el entorno en la estructura organizacional y sistema de gobierno de las universidades?; 2) ¿cuáles son los principales instrumentos de política y gestión de las interacciones con el entorno de las universidades?; y 3) ¡cuáles son las concepciones y valoraciones sobre las interacciones universidad-entorno predominantes entre los funcionarios de gestión universitarios?

Se analizó la estructura organizativa de cada universidad y los instrumentos institucionales orientados a la relación con el entorno territorial, normativas referidas al gobierno universitario, al ingreso y evaluación del personal académico, el tipo de matrícula, la política y gestión de la relación con el entorno. Asimismo, se llevó adelante un análisis interpretativo de 22 entrevistas a informantes calificados que buscó conocer la valoración que los funcionarios universitarios tienen sobre el rol de la universidad en el entorno, y sus concepciones sobre dicho entorno - cómo conciben la matrícula estudiantil, a los otros actores con los cuales la universidad se relaciona o debería relacionarse, y al alcance territorial del entorno de influencia de la universidad-. Los funcionarios entrevistados fueron seleccionados atendiendo a su función y cargo: máximas autoridades universitarias y funcionarios a cargo de las áreas responsables de la vinculación con el medio. Todas las personas entrevistadas fueron debidamente informadas sobre los objetivos de la investigación y prestaron consentimiento personal e institucional para grabar el audio de la entrevista y publicar los resultados. La cantidad de entrevistas realizadas por universidad responde 
a un criterio de saturación teórica. Las citas provenientes de entrevistas fueron anonimizadas.

Tabla 1. Entrevistas según pertenencia institucional y nivel jerárquico de los entrevistados

\begin{tabular}{|c|c|c|c|}
\hline $\begin{array}{l}\text { Entre- } \\
\text { vistados/ } \\
\text { Universidad }\end{array}$ & $\begin{array}{l}\text { Rector, } \\
\text { Vice- } \\
\text { rrector, } \\
\text { Secretarios }\end{array}$ & $\begin{array}{l}\text { Directores } \\
\text { de centros } \\
\text { de investiga- } \\
\text { ción }\end{array}$ & $\begin{array}{l}\text { Investi- } \\
\text { gadores } \\
\text { a tiempo } \\
\text { completo }\end{array}$ \\
\hline $\begin{array}{c}\text { Universidad } \\
\text { Nacional del } \\
\text { Sur (UNS) }\end{array}$ & 2 & & 1 \\
\hline $\begin{array}{c}\text { Universidad } \\
\text { Nacional } \\
\text { Arturo } \\
\text { Jauretche } \\
\text { (UNAJ) }\end{array}$ & 2 & 4 & \\
\hline $\begin{array}{c}\text { Universidad } \\
\text { Nacional } \\
\text { de Quilmes } \\
\text { (UNQ) }\end{array}$ & 4 & 1 & \\
\hline $\begin{array}{c}\text { Universidad } \\
\text { Nacional } \\
\text { de Mar } \\
\text { del Plata } \\
\text { (UNMDP) }\end{array}$ & 5 & 1 & 2 \\
\hline
\end{tabular}

Fuente: elaboración propia.

\section{Resultados}

Esta sección se organiza de la siguiente forma: 1) breve caracterización de las universidades y sus entornos geográficos; 2) análisis de instrumentos institucionales y discursos del staff universitario y 3) análisis comparado de los hallazgos encontrados.

\subsection{Las Universidades Nacionales del Sur, Mar del Plata, Quilmes y Arturo Jauretche}

En este apartado se caracterizan sucintamente las universidades y sus entornos geográficos usando datos aportados por el Instituto Nacional de Estadís- ticas y Censo (INDEC) y por el índice de calidad de vida elaborado por investigadores del Consejo Nacional de Investigaciones Científicas y Técnicas (CONICET) de Argentina (CONICET, Mapa del Índice de Calidad de Vida (ICV), en línea)

\section{Universidad Nacional del Sur}

La UNS fue creada mediante el decreto-ley Nro. 154 el 5 de enero de 1956. Está ubicada en el partido de Bahía Blanca, provincia de Buenos Aires, que posee unos 300.000 habitantes y es considerado un nodo regional debido a su amplia oferta de servicios y redes de conectividad. El grueso de su producto bruto geográfico (PBG) está orientado a los sectores manufacturero, petroquímico, comercial y de servicios diversificados. Posee un índice de pobreza del 24 $\%$ y un índice de calidad de vida de 7.10 puntos. La UNS actualmente es una universidad de tamaño mediano, con una matrícula de alrededor de 30.000 alumnos activos en los niveles de pregrado y grado.

\section{Universidad Nacional de Mar del Plata}

La UNMDP fue creada en el año 1975 con la promulgación de la Ley 21.139 a partir de la nacionalización de dos universidades preexistentes: la Universidad de la Provincia de Buenos Aires y la Universidad Católica Stella Maris, que habían sido creadas respectivamente en los años 1961 y 1958.

La UNMDP se ubica en el partido de General Pueyrredón, que tiene una población de 619.000 habitantes y aporta el 1,6 \% del PBI del país y el $4,5 \%$ del PBI de la Provincia de Buenos Aires (UNMDP, Facultad de Ciencias Económicas y Sociales Producto Bruto Geográfico del Partido de Gral. Pueyrredón, en línea ). La universidad tiene su sede en la ciudad de Mar del Plata, que constituye el principal núcleo poblacional y productivo del sudeste provincial. Posee un índice de pobreza, del 29,5 \% y un índice de calidad de vida de 7.60. La economía del partido se encuentra fuertemente orientada hacia el sector servicios, dado que Mar del Plata constituye 
el principal destino turístico del país. A su vez, son de importancia el cinturón frutihortícola y la industria pesquera, primera en importancia en el país. La UNMDP es una universidad de un tamaño medio, con una matrícula de 30.300 alumnos (año 2016).

\section{Universidad Nacional de Quilmes}

La UNQ fue creada mediante la Ley No 23.749 el 29 de septiembre de 1989. Se ubica en el Partido de Quilmes, que tiene una población de 580.000 habitantes y se sitúa al sur del segundo cordón del conurbano (zona metropolitana que rodea a la ciudad de Buenos Aires, capital de Argentina). Se trata de una región con las características propias derivadas de una alta densidad poblacional —según el censo 2010 albergaba a casi 10 millones de habitantes, de una población total del país de alrededor de 41 millones- y elevados índices de pobreza (alrededor del $35 \%$ para el año 2019). Por su parte, el Partido de Quilmes posee un índice de pobreza del 39,8 \% y un índice de calidad de vida de 6.49 puntos. El PBG del partido está fuertemente orientado a distintas ramas de la industria manufacturera. La UNQ posee un tamaño mediano, con una matrícula de alrededor de 25.000 alumnos regulares en la modalidad presencial más unos 6.000 en la modalidad virtual.

\section{Universidad Nacional Arturo Jauretche}

La UNAJ fue creada el 2 de diciembre de 2009 (Ley 26576) en el partido de Florencio Varela, en la región conurbano sur. El partido posee 425.000 habitantes y su PBG está muy vinculado a la producción flori-hortícola y a la industria manufacturera. El índice de pobreza del partido es del 39,8 \% y el índice de calidad de vida de 5.53 puntos. Las características sociodemográficas más destacadas de esta región son la concentración y el crecimiento poblacional, el déficit habitacional, la contaminación ambiental y la pobreza. La UNAJ posee actualmente un tamaño medio, con una matrícula de alrededor de 20.000 alumnos regulares.

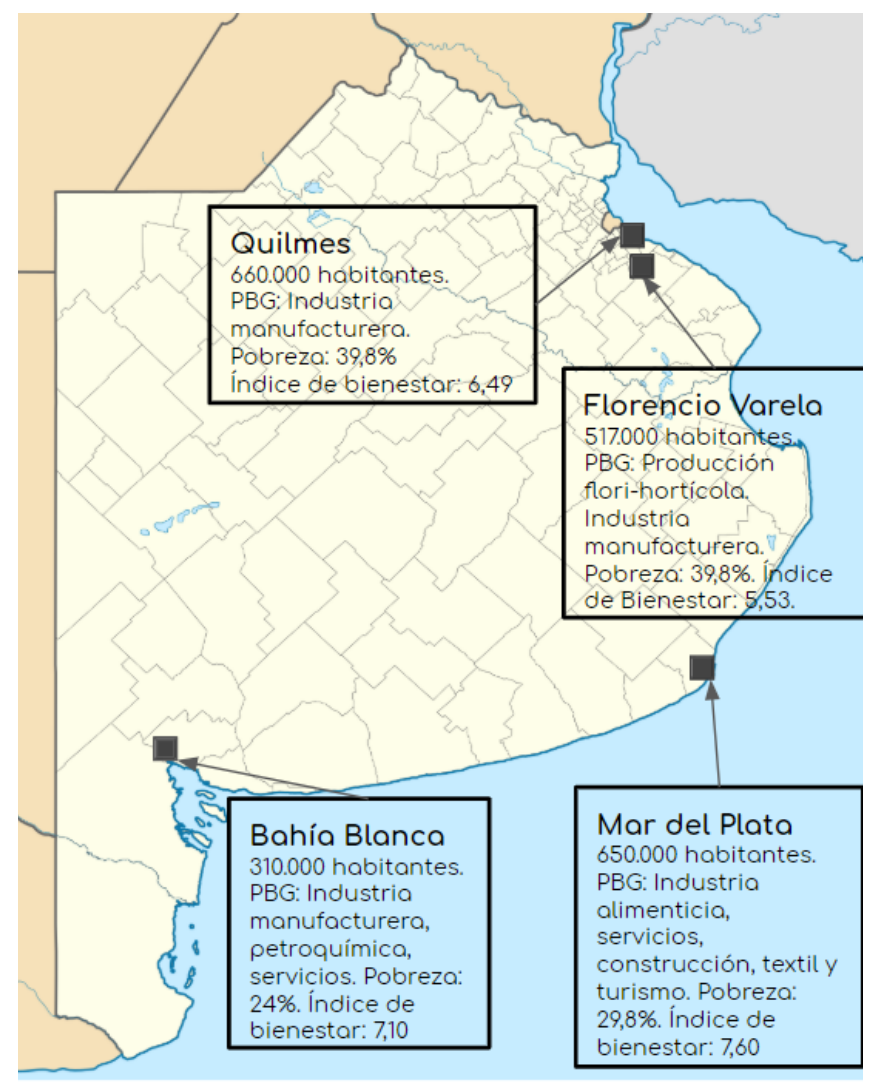

Figura 1. Principales datos socio-económicos de los partidos de la provincia de Buenos Aires donde se asientan las universidades

Fuente: elaboración propia con base en datos INDEC, ICV-CONICET.

\subsection{Modalidades de interacción con el entorno: instrumentos institucionales y discursos}

El lugar de las interacciones con el entorno en la estructura organizacional y gobierno de las universidades

Los espacios organizacionales que concentran las funciones de relación con el entorno socio-productivo en las universidades argentinas son casi exclusivamente aquellos encargados de las funciones de extensión y vinculación/transferencia. En este apartado se describe la estructura que cada universidad adopta para estas funciones y su jerarquía organizacional y los espacios diseñados para permitir la participación de actores extra académicos en las decisiones de gobierno universitario. 
Excepto UNAJ, todas las universidades realizan una división organizacional y funcional entre las tareas de extensión y las de vinculación/transferencia. En UNQ, UNS y UNMDP la función de extensión tiene rango de secretaría. La función de vinculación/ transferencia tiene rango de secretaría en UNQ y rango de subsecretaría en UNS y UNMDP. UNAJ presenta un tipo de organización diferente, que nuclea el vínculo con el entorno en un solo espacio organizacional: el Centro de Política y Territorio (CPT), estructura que posee un rango equivalente a una secretaría. El CPT está organizado en Unidades y Coordinaciones y cuenta con las Unidades de Vinculación Cultural, de Vinculación Tecnológica, de Vinculación Audiovisual y de Vinculación Educativa.

En UNS la extensión universitaria se lleva adelante desde la Secretaría General de Cultura y Extensión Universitaria (SGCyEU) y en particular desde la Subsecretaría de Extensión Universitaria (creada en 2019). Esta actividad fue jerarquizada en el ańo 2007, dado que antes estaba subsumida en la Secretaría General de Relaciones Institucionales y Extensión Universitaria (Res.CSU-46/07). En la UNMDP la extensión se gestiona desde la Secretaría de Extensión Universitaria (SEU). El mismo nombre lleva el ámbito de gestión de la extensión en la UNQ. Esta Secretaría se compone de la Dirección General de Extensión y la Dirección General de Vinculación Social. En estas dos últimas universidades, el área de extensión ha crecido en los últimos años en cantidad de personal y de incorporación de nuevas áreas. Por ejemplo, en la UNQ se sumó en 2013 el Programa Universitario de Incubación Social (PUIS) y en la UNMDP desde 2012 la secretaría sumó la coordinación del Programa de Prácticas Socio Comunitarias mediante la tarea de la formación de docentes y graduados que acompañen las prácticas estudiantiles y desde 2013 la coordinación de los Centros de Extensión Universitaria.

La función de vinculación en UNS es ámbito de la Subsecretaría de Vinculación Tecnológica (SVT) depende de la Secretaría General de Ciencia y Tecnología, que incluye también la gestión de las activi- dades de investigación. Este espacio también fue jerarquizado en 2007 y está compuesto por un área de propiedad intelectual y transferencia, un área de emprendedorismo y un área de asistencia administrativa. En la UNMDP la función de vinculación y transferencia se gestiona desde la Subsecretaría de Transferencia y de Vinculación Tecnológica (STVT), la cual es Unidad de Vinculación Tecnológica (UVT) en el marco de la Ley 23877 de 1991 que habilitó esta figura. La Secretaría de Innovación y Transferencia Tecnológica (SITTEC) de la UNQ fue creada en el año 2012; anteriormente formaba parte de la estructura de la Secretaría de Investigación bajo de denominación de Dirección de Vinculación y Transferencia Tecnológica (DVTT). Desde su jerarquización, su personal se triplicó. Se encarga de centralizar las tareas de valorización y transferencia de la $\mathrm{I}+\mathrm{D}$ de la universidad.

La UNS posee la Fundación de la Universidad Nacional del Sur (FUNS), creada en 1993 como UVT. Trabaja en articulación con la SVT abocándose principalmente a las cuestiones administrativas, legales y financieras relativas a la prestación de servicios y la administración de proyectos. La universidad fue pionera en la generación de este tipo de estructuras organizacionales para la gestión de la vinculación. En 1979 creó la Fundación del Sur para el Desarrollo Tecnológico (FUNDASUR) con el objetivo de gestionar y administrar desde un organismo externo a la universidad los fondos del Programa de Investigación y Desarrollo del Complejo Petroquímico de Bahía Blanca (PIDCOP).

En relación a la participación de la comunidad en el gobierno universitario, las cuatro universidades poseen Consejos Sociales (CS).

Desde su creación, por estatuto, la UNQ cuenta con un Consejo Social Comunitario, que aporta un consejero en el Consejo Superior con voz, pero sin voto. Sin embargo, esta estructura no posee peso real en las decisiones de gobierno, y su nivel de actividad es bajo.

"Se hacían reuniones dos o tres veces por año, pero nunca se logró la permeabilidad que se espe- 
raba" (Entrevista funcionario UNQ no 5, Comunicación Personal, 27 de agosto de 2019).

Una apreciación similar realizan los funcionarios de UNAJ respecto al Consejo Social. Sin embargo, en este caso, el argumento es que el CS no funciona porque las interacciones con miembros de la comunidad son muy frecuentes, lo que las hace más ágiles que una estructura burocrática; "el Consejo Social no funciona porque vemos a diario a sus integrantes" (Entrevista funcionario UNAJ $n^{\circ} 7$, Comunicación Personal, 27 de junio de 2018).

La UNMDP cuenta con un antecedente de consejo social en el Consejo de Integración Universidad y Sociedad (CINTUS), el cual reunía a los representantes de las cámaras empresariales, del mundo gremial y de otras asociaciones de la sociedad civil. Fue creado en 2010 (Resolución del Rector No1924/10), con el objetivo de constituirse en un espacio de intercambio entre los sectores públicos, privados y de la sociedad civil (Cecchi, et al., 2013). Durante muchos años tuvo un rol muy marginal en relación a las expectativas puestas en su creación. Recién en el año 2019 la UNMDP decidió reactivar una idea similar y lanzar un Consejo Social, el cual contó con la participación de Cámaras Empresariales y Sindicatos. De acuerdo con el Rector de la UNMDP, el CPN Alfredo Lazzeretti (2019):

Buscamos escuchar que es lo que el medio demanda de la universidad, nosotros contar que hacemos, ver qué temas podemos realizar en conjunto. Para la universidad es importante tener en presente esta demanda social, necesitamos conocer qué piensan de la oferta académica, la extensión, los proyectos de investigación. (Segunda convocatoria del Consejo Social de la UNMDP. Canal Universidad de Mar del Plata. En línea.).

En el año 2007 la UNS creó una Comisión Asesora de Planeamiento para avanzar con la elaboración de un plan estratégico de mediano y largo plazo en torno a cinco ejes, uno de los cuales es la "Integración de la universidad con la región de influencia" (Plan estratégico de la Universidad Nacional del Sur: 2011-2016-2026). El Plan estratégico previó la creación de un Consejo Social (CS) que fue concretado en diciembre de 2019 con el objetivo de "construir un ámbito de discusión entre los distintos actores de la comunidad, respecto al desarrollo educativo, social y económico del ámbito local y regional" (Resolución CSU 914/19). No está previsto que los representantes del CS estén involucrados en el gobierno de la universidad ni en la elección de sus autoridades.

\section{Instrumentos de politica y gestión de las interacciones} con el entorno

Nos concentramos en este apartado en los instrumentos que abarcan al conjunto de la universidad, dejando fuera de consideración a aquellos que son propios de facultades o departamentos. De igual forma seleccionamos aquellas acciones e instrumentos de mayor envergadura para la universidad o que comportan una innovación institucional. En este sentido, no consideramos aquí acciones de capacitación académica de actores no universitarios o de difusión de las actividades académicas que son formas "tradicionales" que asumió la relación de la universidad con el medio.

Un aspecto común a las cuatro universidades es la jerarquización de la función de extensión universitaria y el diseño de instrumentos que le imprimen un nuevo tono territorial, que busca diferenciarse del ideal iluminista del movimiento reformista de principios de sXX. Más allá de esta coincidencia, cada institución ha diseñado estrategias diferentes para gestionar la relación con el entorno.

Consideración de la función de extensión/vinculación en el ingreso y permanencia del personal académico

La revalorización de la función de extensión puede observarse a partir de los mecanismos de ingreso y permanencia de los actores académicos en la universidad. La UNQ fue una de las primeras universidades en acreditar las actividades de extensión en la carrera docente. En el año 2005 la universidad modificó su reglamento docente creando tres 
perfiles para el ingreso y permanencia del personal docente: a) docencia e investigación; b) docencia y c) docencia y desarrollo profesional que incluye "el ejercicio profesional en el campo disciplinar de referencia, así como la realización de trabajos de transferencia y desarrollo vinculados con aquél” (Res. CS. 324/05). Luego, en el año 2011, se modificó parcialmente el primer perfil añadiendo la función de desarrollo y se sustituyó el perfil "docencia y desarrollo profesional" por el de "docencia y extensión" que comprende actividades de la Universidad en el "medio social de inserción” (Res. CS. 770/11). Recientemente, el reglamento de evaluación docente establece que los ítems a considerar son la formación, la actividad y producción académica y la gestión universitaria. "La actividad académica comprende la docencia, la investigación y el desarrollo, la extensión y transferencia del conocimiento, la producción artística y otras tareas y productos acreditados por la Universidad" (Res. CS. 193/15). La UNAJ incorpora el criterio de perfiles docentes desde su creación admitiendo los perfiles de a) docencia; b) docencia e investigación y c) docencia, vinculación y desempeño profesional (Res. CS. 113/15). En la UNS y UNMDP las actividades de extensión/vinculación no suman puntos para la carrera docente.

\section{Instrumentos orientados a la inclusión educativa}

La inclusión educativa ha sido un tema de creciente interés por la política de educación superior (Marquina y Chiroleu, 2015) y de las universidades.

La UNQ durante los últimos años ha generado espacios institucionales y acciones en el marco de la inclusión educativa. Por ejemplo: la creación en 2015 de una escuela técnica que se instaló en un barrio de bajos recursos del conurbano sur y las acciones destinadas a la retención de la matrícula durante el primer tramo educativo como el Taller de Vida Universitaria, instrumento creado en 2015 destinado a facilitar la transición entre el nivel secundario y la educación superior. Otras acciones en este sentido son las becas de apoyo económico, comedor, material bibliográfico y guardería (beca que también otorga UNAJ).
La inclusión educativa, expresada en instrumentos diseñados para lograr la retención de la matrícula estudiantil, es el aspecto más innovador de la relación con el entorno en la UNAJ. La universidad posee, con un rango equivalente a una facultad, un Instituto de Estudios Iniciales donde se dictan un curso preparatorio para la vida universitaria y cuatro materias introductorias comunes a todas las carreras. Lo novedoso de esta estructura organizacional es que responde al criterio pedagógico de inclusión educativa y no a un nucleamiento disciplinar. Otros instrumentos destacables son programas específicos donde grupos de pedagogos revisan los programas curriculares, los planes de estudio y asisten a clases para detectar problemas en los modos de transmitir conocimientos y/o en la forma de organizar los contenidos. La captación, el ingreso y la retención de la matrícula fue construido como un problema sobre el cual intervenir y ha sido la cuestión que más esfuerzos recibió desde la proyección y los primeros años de funcionamiento de la universidad.

En el caso de la UNMDP la principal iniciativa para fomentar la inclusión educativa es el Programa de Acceso a la Educación Universitaria. Este programa despliega una serie de iniciativas que aspiran a asesorar y acompañar a los alumnos nuevos. Sus objetivos están relacionados con la promoción de la inclusión y permanencia de los aspirantes e ingresantes a la universidad, así como garantizar la inserción de todas las personas a la comunidad universitaria. De acuerdo con la Subsecretaría de extensión (comunicación personal, 10 de julio de 2018):

Nosotros trabajamos con 2 líneas principales que tienen que ver con: aumentar la presencia territorial de la universidad y visibilizar la presencia existente; esto se da principalmente a través de la articulación de todas las iniciativas que se hacen en territorio con los centros de extensión que están distribuidos en los barrios.

La UNS cuenta con el Programa de Tutorías Académicas, el cual es llevado adelante por cada Departamento y está destinado a los estudiantes 
ingresantes, para acompañarlos durante el pasaje de la escuela secundaria a la universidad y reducir la deserción. La universidad otorga subsidios económicos, de transporte y de comedor, "beca bicicleta", —entrega a ciertos estudiantes en concepto de donación una bicicleta y un casco-, y ofrece la posibilidad de alojarse en Residencias Estudiantiles a aquellos estudiantes que provienen de localidades cercanas a la ciudad y cuya situación socioeconómica amerita su otorgamiento.

\section{Articulación con actores del territorio}

Durante los últimos años, las cuatro universidades han intensificado las líneas de acción orientadas al fortalecimiento de la economía social. Ejemplo de ello es el Programa Universitario de Incubación Social de la UNQ, que tiene como objetivo fomentar procesos de fortalecimiento, desarrollo, autonomía y sustentabilidad de la economía social y solidaria (ESS). Actualmente funcionan ocho incubadoras en las áreas de redes de consumidores y productores de la ESS; tecnologías sociales generadas en el marco de la ESS; procesos asociativos y cooperativos para la intervención socioambiental; fortalecimiento de procesos de diseño y comunicación de asociaciones de le ESS; fortalecimiento de empresas sociales; puesta en valor de producción de las comunidades locales en clave de turismo de base comunitaria; generación de capacidades tecnológicas en comunidades rurales, fortalecimiento de prácticas editoriales asociativas.

Desde 2018 el CPT de UNAJ posee una línea de financiamiento "UNAJ Vincula" para proyectos de vinculación "que aporten soluciones a problemas identificados en articulación con actores y organizaciones de la comunidad". Es requisito de este subsidio poseer una contraparte adoptante de los resultados. También la UNS en el año 2007 creó los Programas de Extensión y de Voluntariado de la universidad, que tienen como requisito articular con alguna organización social, institución u organismo gubernamental para llevar adelante los proyectos.

Por su parte, la UNMDP creó el Programa de Vinculación Socio Productiva (PVSP) que busca promover el desarrollo de emprendimientos productivos en los que se trabaje de manera asociativa, ya sea bajo la figura de cooperativas o de ferias. Otra política destacada de la Secretaría de extensión de UNMDP es la generación de Centros de Extensión Universitaria (CEU) en sedes barriales.

"Se busca fortalecer su presencia en el territorio. Esos espacios tienen como principal finalidad hacer de la universidad una idea posible para comunidades que hoy mayormente no acceden a ella" (Funcionario UNMDP $\mathrm{n}^{\circ}$ 5, comunicación personal, 10 de julio de 2018).

La SITTEC UNQ cuenta desde 2013 con una línea de subsidio propia, orientada a la Promoción de Proyectos de Potencial Transferencia Tecnológica (SPOTT). En 2018 la universidad inició el Programa de Apoyo a Proyectos de Desarrollo Tecnológico e Innovación (ProDeTei), con el fin de identificar y fortalecer proyectos con valor tecnológico y/o comercial generados en la universidad. La universidad también cuenta con un Programa de Emprendedores que asesora en las distintas etapas de un proyecto, de cara a su valorización en el mercado.

La STVT UNMDP despliega una serie de iniciativas entre las que destaca el Centro de Apoyo a la Transferencia de Tecnología (CATTEC), de reciente creación y radicado en el Parque Industrial General Savio. Entre las principales actividades que realiza el CATTEC figuran la presentación de capacidades en servicios tecnológicos de la UNMDP para llevar adelante actividades con empresas, relevamiento de necesidades y demandas tecnológicas, capacitación y promoción de los programas de becas y prácticas profesionales. Otro espacio de relación con el entorno desde la STVT es la Incubadora de Empresas de Base Tecnológica e Industriales, creada a fines de 2015.

En relación con los vínculos con los sectores productivos, una característica de UNAJ es actuar como un espacio sede, un nodo articulador de distintos actores del sector industrial de la región (casi todos PYME). Las tareas de articulación están a cargo de la UVT y suplen a las acciones de valorización de proyectos debido a que UNAJ no cuenta aún 
con una masa crítica de actividades de investigación y desarrollo.

En términos de articulación territorial UNAJ forma parte del centro público privado Centro Tecnológico Metalúrgico (CETEM), donde participa también la Asociación de Industriales Metalúrgicos de la República Argentina. Está ubicado en el parque industrial de Florencio Varela y su objetivo es "promover el desarrollo de la industria metalúrgica mediante la realización de actividades académicas y de investigación orientadas a la innovación de los procesos productivos y al perfeccionamiento tecnológico del sector" (Centro Tecnológico Metalúrgico, en línea). Pero en este tipo de iniciativas es la UNS la que tiene mayor trayectoria, al estar involucrada o liderar el desarrollo de polos tecnológicos y parques científicos y tecnológicos. Por ejemplo, el Polo Petroquímico y la UNS mantuvieron una intensa vinculación desde sus orígenes a través de uno de sus institutos de investigación, PLAPIQUI, que desarrolló actividades de investigación y desarrollo, asistencia técnica y de capacitación para las empresas. Por otra parte, desde el Instituto de Investigaciones en Ingeniería Eléctrica se impulsó la creación, en 2010, de Tecnópolis del Sur, un polo científico y tecnológico en el área de microelectrónica de carácter público privado, donde también participan la Unión Industrial de Bahía Blanca y al Instituto Nacional de Tecnología Industrial (INTI).

UNAJ posee una fuerte articulación con la política local, que es reforzada porque una porción significativa de su planta docente se compone de personal que ejerce, asimismo, como funcionario municipal. Las áreas con mayor vinculación son: ambiente e industria. La Secretaría de Industria de Florencia Varela fue creada por profesionales de la universidad por petición del municipio. Dentro de su estructura opera el Instituto de Desarrollo Local (IDEL), donde funciona un banco de proyectos productivos innovadores que, de acuerdo con su grado de viabilidad, son transferidos a la UVT de UNAJ que busca alumnos avanzados que los desarrollen.
Inclusión en las currículas de las actividades de vinculación/extensión

En este ítem se destaca la UNMDP a partir del Programa Universitario de Prácticas Socio Comunitarias mediante el cual la universidad ha iniciado un proceso de curricularización de las prácticas sociocomunitarias en todas las carreras de la misma. Si bien poseen prácticas preprofesionales en algunas carreras, el resto de las universidades no ha impulsado su inclusión masiva en la currícula.

Concepciones del entorno y valoraciones de la interacción universidad-entorno

Como rasgo común, en las entrevistas realizadas a funcionarios de las cuatro universidades existe una valoración positiva de las interacciones de la universidad con el entorno.

La diferenciación organizacional entre las funciones de extensión y transferencia/vinculación que describimos al comienzo en la UNQ, UNS y UNMDP se estructura con base en concepciones bien distintas respecto a la función social de la universidad, los paradigmas a partir de los cuales interactuar con su entorno y qué grupos sociales debe priorizar en tales interacciones.

Por el contrario, en UNAJ existe no solo una estructura organizacional que centraliza la gestión de las relaciones con el medio - productivo, social, cultural y político- , sino un discurso unificado que se articula alrededor de la idea de que la universidad "es parte" del entorno. Esto significa que no busca al entorno mediante acciones vinculantes, sino que se imbrica en él fundamentalmente porque su matrícula está conformada casi exclusivamente por estudiantes locales.

“... el entorno aparece porque los estudiantes traen sus problemas sociales, laborales, etc (...)" (Entrevista funcionario UNAJ no 7. Comunicación personal, 27 de junio de 2018).

“... nuestra concepción no es 'abrirse a' nosotros 'somos parte de' (...)" (Entrevista funcionario UNAJ $\mathrm{n}^{\circ} 2$. Comunicación personal, 2 de julio de 2018) 
En la UNMDP desde extensión, se privilegian las relaciones con asociaciones civiles, principalmente a través de los centros de extensión territoriales y las prácticas sociocomunitarias. Al contrario, las funciones de transferencia y vinculación tecnológica se orientan conceptualmente por el "emprendedorismo", y sus interlocutores son fundamentalmente empresas. También en la UNQ y UNS hay una división marcada entre las áreas de extensión y vinculación de acuerdo con los actores del entorno con los que interaccionan. Extensión se vincula con actores de la economía social y asociaciones civiles, mientras que vinculación lo hace con el sector productivo.

Otro rasgo compartido en el discurso de los funcionarios de gestión de las universidades analizadas es la utilización de marcos interpretativos para definir al trabajo de extensión que se contraponen al asistencialismo o al iluminismo de corte reformista.

El marco conceptual de abordaje es la perspectiva crítica (no iluminista) de la extensión; las intervenciones a través de las prácticas comunitarias deben tensionar, generar reflexiones sobre lo estatuido. (....) Buscamos tensionar los sentidos de las universidades; las prácticas no son sólo intervenciones instrumentales; detrás de ellas está la búsqueda de la transformación social y política. (Entrevista funcionario UNMDP no 6. Comunicación personal, 14 de noviembre de 2019)

"No nos gusta el término extensión. No hay que ir a buscar al pueblo. Abrimos las puertas y se llena de pueblo" (Entrevista funcionario UNAJ no 6. Comunicación personal, 3 de mayo de 2018)

La propuesta nuestra es lo de la construcción colectiva, nosotros, los universitarios, no vamos con una propuesta y la bajamos donde nos parece, sino la idea es ver, a partir de las demandas, de las problemáticas y de las necesidades, qué podemos construir juntos. (Entrevista funcionario UNS no 2. Comunicación personal, 10 de noviembre de 2017).
Para el área de vinculación, en las cuatro universidades se observó la vigencia de un discurso que revela una visión interactiva de producción de conocimientos. "Se invoca la idea de que la demanda no está definida de forma clara por los actores externos, sino que, al contrario, termina de configurarse con la intervención de la universidad". (Di Bello y Romero, 2018b, p.167)

En términos generales, cuando los funcionarios hablan de entorno se refieren a un territorio definido. En el caso de la UNAJ el recorte espacial está claramente delimitado a la región conurbano sur. En los otros casos esto varía según el área de gestión: en el área de extensión, en general, el recorte espacial es más acotado al municipio o región donde se asienta la universidad, mientras que en el ámbito de la vinculación los límites del entorno son más difusos, porque no siempre se expresan en términos territoriales - en ocasiones refieren a sectores o ramas productivas-. Por ejemplo, en la UNQ la noción de entorno que prevalece para el área de vinculación "responde a la idea de un sistema regional de innovación por donde circulan flujos de conocimiento entre diferentes instituciones en las cuales agentes con capacidades disímiles intercambian conocimientos en pos de valorizarlos de cara al mercado" (Di Bello y Romero, 2018b, p.161). Por su parte, en la UNS esta territorialización de la institución y de sus prácticas en su entorno inmediato se observa, principalmente, mediante la modalidad de participación en Polos Tecnológicos, científicos e industriales. En lo que se refiere a las actividades culturales y de extensión de la UNS, la región de influencia queda asociada a la región del sudoeste de la provincia de Buenos Aires, lo que se denomina "región local de influencia" (Plan estratégico de la Universidad Nacional del Sur: 2011 2016-2026).

Si observamos la relación con el entorno en términos de la matrícula, de quiénes son los destinatarios de las acciones de docencia, encontramos diferencias entre las universidades estudiadas.

En la UNMDP tiene fuerza un discurso de reivindicación y de identidad institucional en relación con 
la implementación de las prácticas sociocomunitarias. En este discurso, la preparación práctica y con conciencia solidaria de los alumnos y la responsabilidad social de la universidad pública constituyen su sentido. Mediante los Centros de Extensión Universitaria, la UNMDP manifiesta querer llegar a los estudiantes con dificultades para acceder a la educación superior por barreras socioculturales antes que logísticas.

Tanto en la UNQ como la UNAJ funcionan actualmente instrumentos de retención de la matrícula, aunque la diferencia es que esto no fue una marca de origen en la UNQ mientras que, desde su creación, UNAJ hizo de la inclusión educativa una marca institucional. Esa diferencia se nota, fundamentalmente, en la cultura académica, dado que en UNAJ el proceso de reclutamiento del personal académico tuvo en cuenta, en gran medida, un "perfil comprometido" y alineado con los valores institucionales de la universidad.

"Los profesores están comprometidos con los sectores populares. Si no, no vienen" (Entrevista funcionario UNAJ $n^{\circ} 2$. Comunicación personal, 2 de julio de 2018).

\section{Conclusiones}

En concordancia con los procesos señalados por la literatura internacional y regional sobre las transformaciones en las interacciones universidad-entorno, en las universidades estudiadas se observan cambios recientes que otorgan una mayor envergadura institucional a las interacciones con el medio social y productivo.

Un aspecto común a los cuatro casos es la jerarquización, en los últimos años, de los espacios organizacionales encargados de gestionar las relaciones con el entorno. Este proceso se manifiesta en la promoción del rango que ocupan esos espacios en la organización y/o en un aumento del volumen e importancia de las funciones y del personal afectado a esas tareas.

Las cuatro universidades cuentan con la figura del Consejo Social, como espacio de intercambio con actores de la comunidad y, en UNQ y UNAJ, también como un actor que participa en los órganos de gobierno de la universidad con voz, pero sin voto. Sin embargo, por motivos diversos, en los tres casos donde el Consejo Social está operativo (en UNS es una estructura muy reciente) existen dificultades para lograr que este instrumento sea un mecanismo eficaz para la canalización de las demandas de los representantes de la comunidad.

Asimismo, todas las universidades analizadas han generado recientemente políticas e instrumentos novedosos para gestionar la relación con el medio. Con matices y distinto alcance, según cada institución, sobresalen aquellos diseñados para promover el fortalecimiento de actores de la economía social de la región de influencia de la universidad y las acciones orientadas a la inclusión educativa. Así, a diferencia de lo sucedido en décadas previas, donde los esfuerzos de diseño institucional para la interacción con el medio se concentraban en la relación con las firmas privadas y el sector productivo (Lundvall, 1992; Nelson, 1993; Gibbons et al., 1997, Etzkowitz y Leydesdorff, 1997; Slaughter y Leslie, 1997; Clark, 1998) actualmente se abre paso también a una creciente creatividad institucional para promover las relaciones con un conjunto de actores más amplio (Laredo, 2007; Kruss et al., 2012; Arocena y Sutz, 2015), especialmente con sectores vulnerables de la sociedad y de la trama productiva de la región donde se asientan las universidades, proveyendo recursos y capacidades para resolver problemas específicos del entorno (Gunasekara, 2006) y articulando con usuarios de conocimientos (Arnkil et al., 2010).

Otro rasgo compartido por los cuatro casos - que va en línea con el proceso de jerarquización - es la existencia entre los funcionarios de gestión universitaria de un discurso favorable hacia las actividades de relación con el entorno, y un consenso sobre un ideal de universidad como institución, que ejerce un rol activo en procesos de transformación social en el territorio.

Cabe resaltar, que solo UNAJ presenta mayor homogeneidad - y esto está relacionado con su diseño institucional, con el contexto histórico en el cual fue creada y con su forma centralizada de gobierno- 
respecto de su compromiso regional entre todos los componentes que conforman su comunidad y entre todas las funciones sustantivas de la universidad. En el resto de los casos, la promoción de los vínculos e intervención territorial de la universidad es más reciente, y convive con otras formas e ideales de relacionamiento sedimentados a lo largo de sus trayectorias institucionales.

Los incentivos institucionales para implicar a una mayor proporción de la comunidad universitaria en acciones de articulación con el entorno varían según cada universidad. En la UNQ, por ejemplo, se implementó un sistema de perfiles para ingreso, permanencia y promoción del personal académico que admite las tareas de extensión y vinculación, mientras que en UNMDP el mecanismo que destaca en este sentido es la curricularización de prácticas socio-comunitarias en todas las carreras de grado.

La modalidad predominante que asume la territorialización de las actividades de interacción con el medio que se pudo identificar, de forma preliminar, asume tres modos: a) la universidad como sede; b) la participación en polos científico-tecnológicos y c) el establecimiento de nodos. El primer modo se verifica sobre todo en UNAJ, pero también en UNQ, donde las universidades actúan como sedes, como espacios físicos de reunión de colectivos sociales de la zona. El segundo modo está presente principalmente en UNS, que es una universidad que posee una amplia experiencia en participar de aglomeraciones industriales y científico-tecnológicas con otros actores regionales. Por último, la tercera modalidad tiene lugar fundamentalmente en la UNMDP con la generación de centros de acción territorial en distintas zonas de su región de influencia a partir de los cuales se articulan demandas y acciones con la comunidad.

\section{Referencias}

Appe, S., Rubaii, N., Lippez de Castro, S. y Capobianco, S. (2017). The concept and context of the engaged university in the Global South: Lessons from Latin America to guide a research Agenda. Journal of Higher Education Outreach and Engagement, 21(2), 7-34. Georgia: University of Georgia.

Arbo, P. y Benneworth, P. (2007). Understanding the Regional Contribution of Higher Education Institutions: A Literature Review, OECD Education Working Papers, No. 9, OECD Publishing

Arnkil, R., Järvensivu, A., Koski, P., \& Piirainen, T. (2010). Exploring quadruple helix outlining user-oriented innovation models. Tampere: Tampereen Yliopistopaino Oy Juvenes Print.

Arocena, R.; Sutz, J. (2015). Challenges for Public Higher Education in Uruguay. International Higher Education, (19). Doi: https://doi. org/10.6017/ihe.2000.19.6873

Benneworth, P., Pinheiro, R. and Sánchez-Barrioluengo, M. (2016). One size does not fit all! New perspectives on the university in the social knowledge economy. Science and Public Policy, 43(6), 731-735. Doi: 10.1093/scipol/ scw018

Cecchi, N.; Pérez, D.A.; Sanllorenti, P. (2013). Compromiso social universitario. De la Universidad posible a la Universidad necesaria. Buenos Aires: IEC-CONADU.

Centro Tecnológico Metalúrgico (CETEM). ¿Qué es el Centro Tecnológico Metalúrgico? Recuperado el 15 de frebrero de 2020 de http://www. cetem.org.ar/

Clark, B. (1998). Creating Entrepreneurial Universities: Organizational Pathways of Transformation. Oxford: Pergamon-Elsevier.

CONICET. Mapa del Índice de Calidad de Vida (ICV). Recuperado de https://icv.conicet.gov. ar/

Decreto-ley No 154 (1956). Decreto-Ley de creación de la Universidad Nacional del Sur.

Di Bello, M. y Romero, L. (2018) Concepciones y orientaciones de acción de grupos de investigación académicos sobre sus entornos. Elementos motivacionales, políticos, disciplinares e institucionales. Revista de la Educación Superior. 47 (186) 137-162. 
Di Bello, M. y Romero, L. (2018b) Vinculación y extensión universitaria: la relación entre la universidad y sus entornos en las universidades nacionales de Quilmes y Lanús. Revista Apuntes: revista de ciencias sociales. XVL(82) 145-171. ISSN 0252-1865.

Dosi, G. (1999). Some Notes in National Systems of Innovation and Production and their Implications for Economic Analysis. En: Archibugi, D, J. Howells y J. Michie (Ed.). Innovation Policy in a Global Economy, Cambridge, Cambridge University Press, pp. 35-48

Edquist, Ch. (1997). Systems of innovation approaches. Their emergence and characteristics. In Edquist, Ch (ed.). Systems of Innovation. Technologies, institutions and organizations. Londres: Ed. Pinter.

Enders, J., de Boer, H. F. y Westerheijden, D. F. (2011). Reform of Higher Education in Europe. Rotterdam: Sense Publishers.

Etzkowitz H. y Leydesdorff, L. (2000). The dynamics of innovation: From national systems and "Mode 2" to a triple helix of university-industry-government relations. Research Policy 29(2), 109-123. Elsevier. Doi: https://doi. org/10.1016/S0048-7333(99)00055-4

Fernández Lamarra, N. (comp). (2009) Universidad, Sociedad e Innovación. Una perspectiva internacional, Caseros: UNTREF.

Gál, Z.y Zsibók, Z. (2011). Regional Engagement of Mid-Range Universities: Adapting European Models and Best Practices in Hungary. AUDEM: The International Journal of Higher Education and Democracy (2). Nueva York: State University of New York Press. ISSN 21578591.

García Guadilla, C. (2010). Heterogeneidad y concentración en las dinámicas geopolíticos del conocimiento académico. Reflexiones y preguntas para el caso de América Latina en Mollis, M. et al., Políticas de postgrado y conocimiento público en América Latina y el Caribe, Buenos Aires: CLACSO
Gentile, P. y Levy, B. (2005) Espacio público y privatización del conocimiento Estudios sobre políticas universitarias en América Latina, Buenos Aires: CLACSO.

Gibbons, M., Limoges, C., Nowotny, H., Schwartzman, S., Scott, P. y Trow, M. (1994). The new production of knowledge: The dynamics of science and research in contemporary societies. Thousand Oaks, Estados Unidos: Sage Publications Inc.

Goddard, J. B. y Chatterton, P. (2003). The Response of Universities to Regional Needs. En F. Boekema, E. Kuypers, R. Rutten (eds.). Economic Geography of Higher Education: Knowledge, Infrastructure and Learning Regions, Londres: Routledge.

Goddard J., Coombes, M., L. Kempton, L. y Vallance, P. (2014). Universities as anchor institutions in cities in a turbulent funding environment: vulnerable institutions and vulnerable places in England. Cambridge Journal of Regions, Economy and Society 7(2), 307-325. Cambridge: Cambridge University Press.

Goddard, J., Hazelkorn, E., Kempton, L. y Vallance, P. (2016). The Civic University - The Policy and Leadership Challenges. Chaltenham: Elgar. Doi: $10.4337 / 9781784717728$.

Gunasekara, C. (2006). Reframing the Role of Universities in the Development of Regional Innovation Systems. The Journal of Technology Transfer. Doi: 31. 101-113. 10.1007/s10961005-5016-4.

Guston, D. y Keniston, K. (1994). The fragile contract: University science and the federal government. Cambridge, MA: MIT Press, pp. 270. Doi: https://doi.org/10.1002/pam.4050150221

Hammersley, M. y Atkinson, P. (1994). Etnografía. Métodos de Investigación. Barcelona: Paidós.

Harding, A., Laske, S., Scott, A. (ed.). (2007). Bright Satanic Mills. London: Routledge. Doi: https://doi.org/10.4324/9781315569970

Hoyt, L. y Hollister, R. (2014). Moving Beyond the Ivory Tower: The Expanding Global Movement of Engaged Universities. En B. Hall, y 
R. Tandon, (eds.). Knowledge, Engagement and Higher Education: Rethinking Social Responsibility. Nueva York: Palgrave Macmillan.

Instituto Nacional de Estadísticas y Censos (INDEC). (s. f.). Incidencia de la pobreza y la indigencia en 31 aglomerados urbanos. Primer semestre de 2019. Recuperado de https://www.indec.gob. ar/indec/web/Nivel4-Tema-4-46-152

Facultad de Ciencias Económicas y Sociales. Universidad Nacional de Mar del Plata (2012) Índice de Calidad de Vida. (s. f.). Recuperado de https://www.conicet.gov.ar/?post_type=page\&p $=80364$

Jacob, W.J., Sutin, S.E., Weidman, J.C. y Yeager, J.L. (eds.). (2015). Community Engagement in Higher Education Policy Reforms and Practice. Rotterdam: Sense Publishers. Doi: 10.1007/978-94-6300-007-9

Kruss, G., Visser, M., Aphane, M. y Haupt, G. (2012). Academic Interaction with External Social Partners: Investigating the Contribution of Universities to Economic and Social Development, Ciudad del Cabo: HSRC Press.

Kwiek, M. (2013). Knowledge Production in European Universities States, Markets, and Academic Entrepreneurialism Bern, Switzerland: Peter Lang AG. Series: Higher Education Research and Policy

Lacaze, M. V. et al. (2014). Producto Bruto Geográfico del Partido de General Pueyrredon 20042012; En M. V. Lacaze (coord.). Mar del Plata: Universidad Nacional de Mar del Plata. Recuperado de http://nulan.mdp.edu.ar/2135/1/ PBG_2004-12.pdf

Laredo, P. (2007). Revisiting the Third Mission of Universities: Toward a Renewed Categorization of Universities Activities? Higher Education Policy (20), 441-456.

Lazzeretti, A. (2019). Segunda convocatoria del Consejo Social de la UNMDP. Canal Universidad de Mar del Plata. Recuperado de https:// www.dailymotion.com/video/x7ov4ce
Ley No 21.139. (1975). Ley de creación de la Universidad Nacional de Mar del Plata.

Ley No 23.749. (1989). Ley de creación de la Universidad Nacional de Quilmes.

Ley No 23.877. (1990). Ley de promoción y fomento de la innovación tecnológica.

Ley No 26.576. (2009). Ley de creación de la Universidad Nacional Arturo Jauretche.

Lischetti, M. (coord.) (2013). Universidades latinoamericanas: compromiso, praxis e innovación. Buenos Aires, Editorial de la Facultad de Filosofía y Letras UBA/ Programa de Promoción de la Universidad Argentina, Ministerio de Educación de la Nación, Argentina.

Lundvall, B.A. (1992). National Systems of Innovation: Toward a Theory of Innovation and Interacting Learning. Londres: Pinter Publisher.

Marquina, M. y Chiroleu, A. (2015). Towards a new university map? The expansion of the offer and inclusion as government agenda topics in Argentina. Propuesta Educativa (43), 7-16. ISSN 1995 7785. Buenos Aires: FLACSO Argentina.

Mato, D. (2009). Interculturalidad e inclusión en las universidades. Diferencias culturales, de modos de producción de conocimiento y de modalidades de aprendizaje. En P. Gentili, G Frigotto, R. Leer y F. Stubrin, F. Politicas de privatización, espacio público y educación en América Latina, Rosario CLACSO-HomoSapiens.

Mendizábal, N. (2006). Diseños estructurados y diseños flexibles. En I. Vasilachis. Estrategias de investigación cualitativa. Barcelona: Gedisa. Pp. 65-104

Mollis, M. (2003). Las universidades en América Latina: ¿reformadas o alteradas? La cosmética del poder financiero. Buenos Aires: CLACSO.

Mulgan, G., Tucker, S., Ali, R. y Sanders, B. (2007). Social innovation: what it is, why it matters and how it can be accelerated. Working paper. Said Business School. University of Oxsford. 
Naidorf, J. (2009) Los cambios en la cultura académica de la universidad pública. Buenos Aires, EUDEBA.

Naishtat, F., García Raggio, A.M. y Villavicencio, S. (2001). Filosofías de la universidad y conflicto de racionalidades. Buenos Aires: Colihue.

Neave, G. y van Vught, F. A. (1991). Prometheus Bound: the changing relationship between government and higher education in Western Europe. Oxford: Pergamon Press.

Nelson, R. (ed.). (1993). National Innovation Systems: A Comparative Analysis. New York: Oxford University Press.

Pérez, D.A., Cecchi, N., Lakonich, J.J. y Rotstein, A. (2009). El compromiso social de la universidad latinoamericana del siglo XXI: Entre el debate y la acción. Buenos Aires: IEC-CONADU.

Plan estratégico Universidad Nacional del Sur 20112016 - 2026. (2013). (1a ed.). Bahía Blanca: Editorial de la Universidad Nacional del Sur. Ediuns.

Pinheiro, R., Benneworth, P. \& Jones, G. (eds.). (2012). Universities and Regional Development A critical assessment of tensions and Contradictions. Londres: Routledge.

Resolución del Consejo Superior Universitario 324/05. (2005). [Universidad Nacional de Quilmes].

Resolución del Consejo Superior Universitario 46/07. (2007). [Universidad Nacional del Sur].

Resolución del Consejo Superior Universitario 770/11. (2011). [Universidad Nacional de Quilmes].

Resolución del Consejo Superior Universitario 193/15. (2015). [Universidad Nacional del Sur].

Resolución del Consejo Superior Universitario 113/15. (2015). [Universidad Nacional de Arturo Jauretche].

Resolución del Consejo Superior Universitario 914/19. (2019). [Universidad Nacional del Sur].

Resolución del Rector 1924/10. (2010). [Universidad Nacional de Mar del Plata].
Riquelme, G. (ed.). (2008). Las universidades frente a las demandas sociales y productivas: capacidades de los grupos de docencia e investigación en la producción y circulación de conocimiento. Buenos Aires: Miño y Dávila editores.

Romero, L., Buschini, J., Vaccarezza, L. y Zabala, J.P. (2015). La universidad como agente político en su relación con el entorno: Aproximación teórica metodológica para el estudio de la conformación de vínculos entre la Universidad y su entorno social municipal. Ciencia, Docencia y Tecnología; 26(51), 1-25.

Schugurensky, D. (2007). Higher education restructuring in the era of globalization: Toward a heteronomous model? En R. Arnove, C. A. Torres (eds.). Comparative education: The dialectic of the global and the local. Lanham: Rowman \& Littlefield.

Schwartzman, S. (2008). Universidad y desarrollo en Latinoamérica: experiencias exitosas de centros de investigación. Caracas: UNESCO/IESALC

Slaughter, S. y Leslie, L. (1997). Academic Capitalism: Politics, Policies of the Entrepreneurial University. Baltimore: Johns Hopkins University Press.

Sturniolo, S.A. y Naidorf, J. (2007). Universidad, Investigación y Territorio: ¿Por qué hablar de una Dimensión Territorial en la construcción de conocimiento desde la universidad? Trabajo presentado en el IV Encuentro Nacional $Y I$ Latinoamericano: "La Universidad como Objeto de Investigación. Tucumán: Argentina.

Sutz, J. (1996). Universidad y sectores productivos. Buenos Aires: CEAL.

Storni, A. (2005). Las 'nuevas' universidades del conurbano bonaerense: (10 años después). En $V$ Coloquio internacional sobre la Gestión Universitaria en América del Sur. Mar del Plata: UNESCO.

Tenti Fanfani, E. (1993). La escuela vacía. Deberes del Estado y responsabilidad de la sociedad. Buenos Aires: UNICEF-Losada. 
Tünnermann, C. (2008). Ninety years of the university reform of Córdoba: 1918-2008. Buenos Aires: CLACSO.

Universidad Nacional de Mar del Plata (UNMDP), Facultad de Ciencias Económicas y Sociales, Producto Bruto Geográfico del Partido de Gral. Pueyrredón. Recuperado de https://eco. mdp.edu.ar/pbg/. [Consutado el 13 de febrero de 2020].

Vallaeys, F. (2006). Breve marco teórico de la responsabilidad social universitaria. CD Multimedia, Lima, Perú: Banco Interamericano de Desarrollo. Recuperado de https://www.econo. unlp.edu.ar/frontend/media/86/10986/10ec2d7e617a62eba0696821196efeae.pdf
Vessuri, H. (comp.). (1995). La Academia va al Mercado. Relaciones de cientificos académicos con clientes externos. Caracas: Fondo Editorial FINTEC.

Villa Sanchez, A. (2014). La innovación social en el ámbito universitario: una propuesta para su diagnóstico. Revista Argentina de Educación Superior. (8), 188-218.

Zangrossi, G. (2013). Las universidades del Conurbano Bonaerense, impacto, desafíos y perspectiva, X Jornadas de Sociologia, Facultad de Ciencias Sociales, Universidad de Buenos Aires, Buenos Aires, Argentina. 